Published in final edited form as:

Nat Neurosci. 2019 May 01; 22(5): 778-786. doi:10.1038/s41593-019-0357-8.

\title{
Locomotion-dependent remapping of distributed cortical networks
}

\author{
Kelly B. Clancy ${ }^{\mathrm{iD}, 1,2,{ }^{*}, \text { Ivana Orsolic }}{ }^{1,2}$, Thomas D. Mrsic-Floge $\mathrm{I}^{\mathrm{i} D, 1,2,{ }^{*}}$ \\ ${ }^{1}$ Biozentrum, University of Basel, Basel, Switzerland
}

\begin{abstract}
The interactions between neocortical areas are fluid and state-dependent, but how individual neurons couple to cortex-wide network dynamics remains poorly understood. We correlated the spiking of neurons in primary visual (V1) and retrosplenial (RSP) cortex to activity across dorsal cortex, recorded simultaneously by widefield calcium imaging. Neurons were correlated with distinct and reproducible patterns of activity across the cortical surface; while some fired predominantly with their local area, others coupled to activity in distal areas. The extent of distal coupling was predicted by how strongly neurons correlated with the local network. Changes in brain state triggered by locomotion strengthened affiliations of V1 neurons with higher visual and motor areas, while strengthening distal affiliations of RSP neurons with sensory cortices. Thus, the diverse coupling of individual neurons to cortex-wide activity patterns is restructured by running in an area-specific manner, resulting in a shift in the mode of cortical processing during locomotion.
\end{abstract}

Cortical neurons compute collectively, necessitating flexible cooperation between local and distal networks. Accordingly, they receive input from and provide input to both local and long-range partners ${ }^{1}$. Locally, pyramidal cells in the neocortex make strong and frequent connections with functionally similar partners, and thus belong to subnetworks defined by shared inputs and recurrent connections ${ }^{2-6}$. Notably, the firing of nearby cells is diversely

Kelly B. Clancy: 0000-0002-9877-7840

Thomas D. Mrsic-Flogel: 0000-0002-8947-408X

"Correspondence and requests for materials should be addressed to K.B.C. or T.D.M. k.clancy@ucl.ac.uk; t.mrsicflogel@ucl.ac.uk.

2Present address: Sainsbury Wellcome Centre, University College London, London, UK.

Data availability

All code and data used for these analyses will be made available upon request.

Author contributions

K.B.C. and T.D.M.-F. conceived and planned the experiments, K.B.C. performed the experiments and analyses, I.O. built the widefield microscope, K.B.C. and I.O. designed analyses methods, and K.B.C. and T.D.M.-F. wrote the manuscript.

Competing interests

The authors declare no competing interests.

Reprints and permissions information is available at www.nature.com/reprints.

Journal peer review information: Nature Neuroscience thanks Carsen Stringer, Igor Timofeev, and other anonymous reviewer(s) for their contribution to the peer review of this work.

Publisher's note: Springer Nature remains neutral with regard to jurisdictional claims in published maps and institutional affiliations. 
coupled to ongoing activity in the local network ${ }^{7,8}$, reflecting the degree of synaptic excitation provided by their neighbors ${ }^{8}$. In addition, neurons also connect to others residing in distal cortical areas ${ }^{9-11}$, but little is known about how neurons are yoked to activity in long-range networks ${ }^{8,12-15}$. Recent work in anesthetized animals suggests that a neuron's activity is largely coupled to that of the cortical area in which it resides ${ }^{12}$. A key unknown is whether functional interactions of individual neurons with distal areas become more evident during wakefulness, or whether they shift depending on behavioral state. We therefore undertook to map dorsal-cortex-wide affiliations of neurons in cortical areas with complementary functions: primary visual cortex (V1) and retrosplenial cortex (RSP), an associative area involved in navigation and memory.

Given the diversity of neuronal coupling to the local network, might neurons that are uncorrelated with local activity instead belong to specific long-range ensembles, relaying information from distributed associative networks to shape local computations? Moreover, given the known differences in connectivity patterns of excitatory and inhibitory neural classes, is there a relationship between a cell's identity and its distal affiliations? Parvalbumin-expressing $\left(\mathrm{PV}^{+}\right)$GABAergic inhibitory neurons receive strong and dense excitation from neighboring pyramidal cells, and they are thought to provide feedback inhibition proportional to the activity of the local network ${ }^{16-18}$. Pyramidal cells in rodents, on the other hand, make much sparser connections with their neighbors and are more probable to exhibit independent activity ${ }^{16}$. Are these differences between cell classes evident in their functional coupling to distal networks?

If individual neurons within an area have unique affiliations with activity in local and/or distant cortical areas, these may be dynamic depending on the animal's internal state. Changes in behavioral states are known to influence cortical processing in diverse, areadependent ways ${ }^{19-25}$, but it is unclear whether these state-dependent changes would alter the distal affiliation patterns of individual neurons. Studies using functional magnetic resonance imaging (fMRI) indicate that the coupling between different brain areas is fluid, suggesting that information can be dynamically routed depending on task demands ${ }^{26}$. However, the underlying mechanism remains unresolved because fMRI reflects neural, glial and vascular responses in tissue volumes representing thousands of cells ${ }^{27}$. Specifically, it is unknown whether individual neurons remap their long-range affiliations during different behavioral states, and if so, whether such remapping is unique to cortical areas subserving different functions.

To investigate these questions, we correlated the spiking of individual cortical neurons with simultaneously recorded calcium signals across the dorsal cortex of awake mice. We recorded single units in V1 and RSP, an area thought to match external spatial cues with an animal's internal model during navigation ${ }^{28-30}$. In both areas, we found highly diverse functional affiliations of individual neurons with the rest ofthe dorsal cortex, although the majority of units were predominantly affiliated with the area within which they reside. These affiliations changed depending on the animal's behavioral state: during locomotion, V1 neurons became more locally correlated, while RSP neurons became more correlated with distal sensory areas. Our data raise the possibility that locomotion shifts cortex into a feedforward, sensory-driven processing mode relevant for navigation ${ }^{31}$. 


\section{Results}

\section{Units in V1 and RSP are affiliated with diverse cortical networks}

In order to relate neuronal firing to distal activity patterns, we used multi-channel silicon probes to record spikes from individual neurons while simultaneously imaging calcium signals of the dorsal cortex in transgenic mice expressing the calcium indicator GCaMP6s in $\mathrm{CaMKII}^{+}$pyramidal neurons (see Methods; Fig. 1a; Supplementary Fig. 1). We obtained 755 single units per recording, spanning all cortical layers (see Methods, Supplementary Fig. 2 ) in either primary visual cortex (V1; $N=8$ animals) or dorsal retrosplenial cortex (RSP; $N$ $=11$ animals) in head-fixed mice, which were free to run on a cylindrical treadmill. Mice viewed naturalistic visual stimuli in the contralateral hemifield, interleaved with periods of a blank gray screen. Neurons exhibited a wide range of firing rates, and the most highly active units exhibited the narrow spike waveform characteristic of fast-spiking GABAergic interneurons (Fig. 1b,c).

To quantify the relationship between the firing of individual neurons and global patterns of calcium activity, we computed correlation maps for each recorded unit. Spike trains were aligned and binned to match the times of corresponding imaging frames ( $40 \mathrm{~Hz}$ frame rate), and the correlation coefficient between a unit's spike train and the fluorescence trace in each pixel was used to build a spike-triggered activity map (SpAM) for each unit (Fig. 1d,e; Supplementary Fig. 3). We did not perform hemodynamic correction on the fluorescence signals, as we determined that this does not significantly affect our maps (see Methods, Supplementary Fig. 4). We took the partial correlation with respect to dorsal-cortex-wide activity (mean $\Delta F F$ of all imaged pixels, where $\Delta F F$ is the instantaneous change in fluorescence; see Methods) to remove sources of shared variance (see Methods). In order to compare across subjects, maps were transformed and aligned to a dorsal projection of the common coordinate framework of the Allen Reference Atlas (http://mouse.brain-map.org/ static/atlas) using stereotaxically placed marks on the skull (Fig. 1d,e; see Methods).

We took the sum of $z$-scored single and multi-unit activity from each recording site to build a population correlation map (population SpAM), which revealed the distal activity patterns associated with the net spiking of the local network (Fig. 1f; Supplementary Figs. 3 and 5). As expected from anatomy, the population spiking activity in V1 was most strongly correlated with calcium signals in ipsi- and contralateral visual areas ${ }^{10,11,32}$ and with RSP. Population activity in RSP was most strongly correlated with RSP, anterior cingulate cortex (ACC) and parietal (PTL) and visual areas, consistently with previous studies of the connectivity and activity of areas involved in the default mode network (DMN) ${ }^{10,11,26,33-35}$. The widefield fluorescence signal is a weighted sum of predominantly local dendrites, axons and cell bodies (which are highly correlated; see Methods), and a small proportion of distal axons, which largely reflects local spiking ${ }^{36}$ (Supplementary Figs. 6 and 7).

Nearby units in both V1 and RSP exhibited diverse distributed affiliations (Fig. 1d,e). To quantify their differences from the population average, we calculated the two-dimensional (2D) pixel-by-pixel correlation between each unit's SpAM and the population SpAM. Although many single-unit SpAMs were similar to that of the population in both V1 and RSP cortex, the SpAMs of other neurons were markedly different (Fig. 1g). Specifically, 
individual neurons in both areas were affiliated with distinct patterns of activity across the dorsal cortical surface, including strong coupling to localized regions of the midline prefrontal (ACC), somatosensory and motor cortices (Fig. 1i,j; values $z$-scored to allow visual inspection across units). In some cases, units were more strongly correlated with distal areas than withthe area within which they resided (Fig. 1i,j). To estimate significance, maps calculated using shuffled spike trains were compared with the shuffled population SpAM (Fig. 1g, gray bars; see Methods), revealing that the distribution of the true SpAMs was significantly different than would be expected by chance. Indeed, single-unit SpAMs were robust across interleaved recording epochs, indicating that they reflect reproducible activity correlations (Supplementary Fig. 5; see Methods).

Assuming that different neuronal classes might differ in their coupling to local and distal cortical activity, we categorized units by the shape of their spike waveform. In both V1 and RSP cortex, the SpAMs of fast-spiking units (FS) — putative GABAergic interneuronswere much more similar to the population SpAM than those of regular spiking units (RS, predominantly excitatory neurons; Fig. 1h). This result is consistent with observations that $\mathrm{PV}^{+}$cells track activity of the local network ${ }^{16-18}$, whereas the greater diversity of SpAMs for RS units implies that they are embedded in more diverse long-range subnetworks, as would be expected from previous anatomical studies ${ }^{37}$.

\section{Neurons uncorrelated with the local network have distal affiliations}

We next determined how a neuron's coupling to the local network relates to its coupling to distributed cortical dynamics. Population coupling is a measure of how strongly a given neuron's spiking is yoked to activity within its local network ${ }^{8}$ and is calculated as the zerolag cross-correlation between the spiking of a given cell and the sum of spikes from all other simultaneously recorded, nearby neurons ${ }^{8}$ (Fig. 2a). This metric is related to the relative proportion of local synaptic inputs a neuron receives ${ }^{8}$. We hypothesized that neurons weakly coupled to their local network might instead be more strongly associated with distributed cortical networks. Alternatively, they might reflect coupling to subcortical or neuromodulatory activity. In both V1 and RSP, units that were highly locally coupled had SpAMs more similar to the population SpAM (Fig. 2b-d). In contrast, low-coupled units exhibited the greatest diversity of SpAMs and were less similar to the population SpAM. Notably, SpAMs calculated for data divided across arbitrary epochs were as self-similar for low-coupled as for high-coupled units ( $t$-test for distribution of SpAM self-correlations across interleaved epochs in high vs. low-coupled populations, for V1: $P=0.1, \mathrm{~T}=1.65$, df $=75$; for RSP: $P=0.4, \mathrm{~T}=0.86, \mathrm{df}=37$ ), confirming that the low-coupled units are robustly affiliated with diverse distal patterns. Some low-coupled units were simultaneously affiliated with local and distant activity (for example, bottom unit, Fig. 2b) or anti-correlated with their neighbor's SpAMs (for example, bottom unit, Fig. 2c). Surprisingly, many locally uncoupled cells had high correlations with the population SpAM, suggesting that local mechanisms contribute to their decorrelation (Fig. 2d).

To characterize the cortical activity patterns associated with the firing of high and lowcoupled units, we divided SpAMs based on local population coupling. The cut-offs for lowand high-coupled units were set below the 20th and above the 80th percentiles of the 
normalized coupling coefficients, respectively. The mean SpAMs ( $z$-scored to allow comparison across animals) of low-coupled V1 units reveal that low-coupled units had similar affiliations compared with high-coupled units (Fig. 2e, top row). The pixel-by-pixel variance revealed common affiliation motifs of the two populations: the SpAMs of highcoupled neurons in V1 were very similar to each other, exhibiting low variance, whereas low-coupled neurons exhibited common affiliations with hind limb somatosensory cortex (S1HL), hind limb motor cortex (M1HL), and RSP (Fig. 2e, bottom row). Similarly, lowcoupled neurons in RSP were less correlated with calcium signals in the local network than high-coupled units (Fig. 2f, top row, $t$-test, $P=1 \times 10^{-6}, \mathrm{~T}=5.3$, $\mathrm{df}=84$ ). High-coupled RSP SpAMs were stereotypically similar, exhibiting low variance across the population, whereas the low-coupled units had more diverse affiliation motifs: most commonly visual, somatosensory and motor areas (Fig. 2f, bottom row).

\section{Area-specific, locomotion-dependent switch in distal affiliations}

Locomotion is known to modulate the activity of neurons in an area-dependent manner ${ }^{21,23}$. We hypothesized that locomotion might also change how neurons couple to distributed activity. To test this, we classified our recordings into epochs of quiescence and locomotion (which we defined as periods during which the animal maintained a persistent velocity $>1$ $\mathrm{cm}$ per s for more than $2 \mathrm{~s}$, as previous work has indicated that locomotion-driven dynamics are evident even at this low threshold ${ }^{21}$ ), and calculated SpAMs for both epochs. Mice with total epoch duration of less than 10 minutes for either condition were excluded from the analysis (V1; $N=5$ mice; RSP, $N=5$ mice). Units were diversely affected by running (Fig. $3 a)$. The firing of neurons in V1 became more strongly correlated with calcium signals in visual cortex during locomotion compared with quiescence, irrespective of whether their mean firing rate was enhanced or suppressed by running (Fig. 3b,c; Supplementary Fig. 8). Unlike V1 neurons, however, RSP units were more stereotypically coupled to their local network during quiescence, and became more diversely affiliated during locomotion (Fig. 3d,e; Supplementary Fig. 8). Indeed, locomotion triggered an extensive remapping of SpAMs in RSP, but only to a limited degree in V1 (Fig. 3f; Supplementary Fig. 8), indicating that behavioral state has a profound impact on the distal coupling of RSP cells.

To understand how locomotion affects dorsal-cortex-wide affiliations across assemblies of neurons, we calculated the pixel-by-pixel correlation of each unit's SpAM with those of all other units during quiescence and locomotion. Strikingly, whereas SpAMs of V1 units became more similar to each other during locomotion (Fig. 3g, $P<1 \mathrm{e}-5$ ), the SpAMs of RSP units became dramatically different (Fig. $3 \mathrm{~h}, P<1 \times 10^{-5}$ ) and remapped by locomotion. These changes to distal affiliations of individual cells were observed regardless of whether animals were being shown naturalistic visual stimuli (Fig. 3d,h), in the dark (Supplementary Fig. 9a), or running through a virtual reality corridor (Supplementary Fig. $9 b)$. These data suggest that there is a state-dependent switch that governs how individual neurons couple to distal activity patterns independently of sensory drive. This remapping was not evident between epochs of passive and aroused states in quiescent animals, suggesting that locomotion and arousal have differential effects on cortical dynamics in RSP, as has been shown previously in V1 ${ }^{24}$ (Supplementary Fig. 10). On average, V1 activity was more correlated with visual areas and secondary motor cortex during locomotion, and less 
correlated with somatosensory areas (Fig. 3i,k). RSP activity was more correlated with V1 and somatomotor areas during locomotion than with RSP (Fig. 3j,k), consistently with increased functional coupling with cortical areas engaged by locomotion and sensory feedback. Therefore, locomotion fundamentally reshapes global and local interactions of individual neurons embedded in cortical networks.

Locomotion exerted differential effects on the firing of V1 and RSP neurons. As previously reported, locomotion increased the mean firing rate of V1 units (Fig. 4a, left panel; 26\% increase in regular-spiking units $(P=0.02, \mathrm{~T}=2.2, \mathrm{df}=118, t$-test spike rate during locomotion vs. quiescence) with no significant increase in FS units ${ }^{21,38-41}$ ( $t$-test, $P=0.9$, T $=0.04, \mathrm{df}=9)$. However, running onset induced a large increase in activity in both FS and RS cells in V1, similar to dynamics reported previously ${ }^{24}$ (Supplementary Figs. 11 and 12). In RSP, locomotion modulated the firing of individual regular-spiking units without affecting the mean firing rate ( $t$-test, $P=0.53, \mathrm{~T}=0.6, \mathrm{df}=196$ ) and suppressed a subpopulation of FS units (Fig. 4a, right panel; mean FS suppression 17\%, $t$-test $P=0.02, \mathrm{~T}=2.3$, $\mathrm{df}=37$ ). Using two-photon imaging of virally expressed GCaMP6f in RSP of PV-tdTomato mice, we confirmed that more $\mathrm{PV}^{+}$cells decreased their firing than PV- cells (Supplementary Fig. 12). The activity of superficial units in RSP was more probably increased by locomotion than that of deep units in both spiking and two-photon imaging datasets (Supplementary Fig. 13), consistently with evidence that inputs from motor cortex to RSP preferentially connect with superficial neurons ${ }^{33}$.

In both V1 and RSP, spike correlations dropped during locomotion (Fig. 4b, paired $t$-test, V1: $\left.P=0.02, \mathrm{~T}=2.3, \mathrm{df}=991 ; \mathrm{RSP}: P=1 \times 10^{-40}, \mathrm{~T}=14, \mathrm{df}=3784\right)$, consistently with previous work31 and confirmed by two-photon imaging in RSP (Supplementary Fig. 12f). The drop in spike correlations was marked in RSP and could not be explained by the reduction in firing rate (see Methods). Correlations also decreased during arousal, but not as dramatically as during locomotion, suggesting that different mechanisms under-lie this decorrelation, including the possibility that activity during locomotion may be driven more by distal than by local recurrent inputs compared with quiescence. Indeed, we observed a striking change in the structure of pairwise spiking correlations in RSP but not in V1. In V1, pairs were similarly correlated between quiescence and locomotion, whereas RSP pairs that were highly correlated during quiescence often became less correlated during locomotion, and vice versa (Fig. 4c; Supplementary Fig. 12h,i). Instead, we found that RSP neurons strongly coupled to local population activity were the ones preferentially suppressed by locomotion (Fig. 4d), while weakly coupled RSP neurons were unchanged. There was no relationship between population coupling and firing rate modulation for V1 neurons. Given that the locally low-coupled neurons are those with more diverse distal affiliations, we suggest that locomotion induces a major reorganization of network activity in RSP, wherein a strongly correlated local network is silenced and a more globally coupled network unmasked, perhaps to update RSP representations with sensory information necessary for navigation. Indeed, visual responses in both V1 and RSP were more reliable when the animal was locomoting than when it was quiescent (Supplementary Fig. 14), indicating that locomotion may play a role in shaping how sensory information is shared across the cortex. 
We hypothesized that the FS units suppressed by locomotion might be involved in this stateinduced network switch in RSP cortex, acting to suppress the activity of neurons receiving input from distant areas during quiescence. While the SpAMs of locomotion-suppressed FS cells (units suppressed more than $80 \%$ by locomotion, $N=15$ ) did not qualitatively change between states (Fig. 4e,f), RS units that were significantly anti-correlated with them $(N=$ 13) switched from being anti-correlated with the population SpAM during quiescence to being correlated during locomotion (Fig. $4 \mathrm{e}-\mathrm{g}$ ). Specifically, these RS units became more correlated with activity in visual areas during locomotion (Fig. 4e-g) suggesting that the locomotion-suppressed FS population may gate sensory information routed to RSP, perhaps related to RSP's role in visually guided navigation.

This state-dependent switch in RSP cortex may reflect a shift in affiliations not only with distributed cortical networks but also with subcortical areas invisible to wide-field calcium imaging ${ }^{42}$. The superior colliculus (SC), for example, is a midbrain structure that is bidirectionally connected to $\mathrm{V} 1$ and receives unidirectional projections from RSP, and is known to be involved in orienting behaviors ${ }^{42}$. We recorded spiking activity from neurons in the medial SC and found that they were also dynamically coupled to cortical activity (Supplementary Fig. 15). On average, mSC spiking activity was more correlated with RSP and visual areas during locomotion than during quiescence, suggesting that RSP drive to subcortical areas is strengthened. Thus, the putative switch in the operating regime of RSP also results in a stronger affiliation with the colliculus.

\section{Discussion}

How individual neurons participate in brain-wide network dynamics is currently poorly understood. fMRI studies have yielded important insights into how distant brain areas fluently coordinate their activity despite a static anatomical architecture ${ }^{26}$, but such bulk recording methods preclude mapping these dynamics with cellular resolution. By combining electrophysiological recordings from individual neurons with widefield calcium imaging of dorsal cortex, we identified the affiliations of neural populations in V1 and RSP with multiple regions distributed across the dorsal cortical surface. While most units were affiliated with the area in which they reside-putative FS interneurons almost exclusively so - the activity of many units was also correlated with activations in diverse, distal areas of the neocortex. Importantly, the patterns of local and distal activity associated with a neuron's spiking could be dynamically remapped during locomotion. Of the two areas studied, neurons in RSP exhibited the most dramatic shifts in their affiliations, indicating that they can take part in different functional ensembles in a state-dependent manner. This network restructuring did not happen for all neurons equally—unique patterns emerged for different neurons, indicating that cortical neurons can independently engage in different networks depending on the animal's behavioral state.

\section{Neurons participate in diverse long-range networks}

We found that many cortical neurons correlate with activity in distant cortical areas in addition to, or instead of, their immediate network, in contrast to a previous study undertaken in anesthetized animals that found cortical cells to be largely locally driven ${ }^{12}$. 
Our measure is correlative, and as such, we cannot resolve whether units drive, are driven by, or share latent driving sources with other areas. However, we note that the common motifs of affiliation for V1 and RSP units recapitulated known anatomical inputs to these areas (contra- and ipsilateral visual areas, RSP, ACC, PTL, barrel S1, and S1HL and M1HL) $)^{10,11}$. Therefore, some of these cells might act as 'local representatives' of distal areas, driven more strongly by long-range than by local signals, serving to fold the output of distant networks into local computations. Indeed, recent work indicates that a fraction of neurons in rodent visual cortex are influenced by non-visual signals, suggesting that information is shared across distant brain regions in order to contextualize sensory processing ${ }^{43-47}$. Because S1HL and M1HL are active in locomotion (Supplementary Fig. 3e), affiliations with these areas might only represent the shared latent influence of running. However, many units were correlated with activity in S1HL and M1HL even when animals were stationary, suggesting that locomotion alone cannot account for this affiliation.

\section{Locally uncoupled neurons are affiliated with distal activity}

Responses of most neurons in the neocortex are unreliable and difficult to predict from measurable external or internal variables ${ }^{48,49}$. Correlated activity reflects the organization of recurrent connections ${ }^{2,3}$ and may also arise from shared, task-related or state-dependent signals. In neocortex, however, not all neurons are strongly coactive with their neighbors ${ }^{8}$. Given the preponderance of long-range corticocortical projections, or cortico-thalamocortical pathways, at least some of this response variability may be driven by activity originating in other cortical areas. We expected that neurons weakly coupled to the local population might preferentially participate in and be driven by distributed or distal networks. Indeed, cells least coupled to the local network exhibited the most diverse SpAMs. Some members of this population were still locally affiliated, but many either had long-range affiliations or were generally uncorrelated with the rest of the imaged cortex, potentially representing affiliations with areas invisible to wide-field imaging. Thus, neurons that are uncoupled to local activity often appear to belong to brain-wide ensembles. We speculate that they may integrate the outputs of distant networks and relay them into local computations.

\section{Default mode network}

RSP is known to be involved with spatial navigation and is a major hub of the default mode network (DMN), an evolutionarily conserved system of interacting brain regions that are highly engaged in resting subjects in a state of internal focus ${ }^{26,35}$. In RSP of quiescent animals, units were strongly correlated with activity in RSP, dorsal anterior cingulate cortex and a lateral parietal area (also known as the rostro-lateral visual cortex), in agreement with previous recordings of the DMN in rodents using $\mathrm{FMRI}^{35}$. RSP units were sometimes affiliated with secondary motor areas ${ }^{33}$ and barrel cortex. Weak projections between barrel S1 and RSP have been reported, and given RSP's role in navigation, these could carry information about egomotion relayed by the trident whiskers, or other tactile information that might inform orienting behaviors. Our data suggest that the strong activation of the DMN in resting subjects is related to higher spike rates in an FS population that may act to shunt sensory input from distal cortical areas. 


\section{A switch towards feedforward processing during locomotion}

Cortical neurons simultaneously process multiple streams of information representing not only sensory information but also signals related to motor plans, attention and motivation, in a manner dictated by the animal's current state and objectives ${ }^{20-24}$. Indeed, we found that distal affiliations were dynamically shaped by the animal's behavioral state. The activity of neurons in $\mathrm{V} 1$ became more similar to that of their neighbors and more correlated to activation of visual areas during locomotion, and less coupled to distal regions. We suggest that this reflects a shift in how the visual cortex integrates information towards a feedforward processing regime during locomotion ${ }^{31}$, thus giving greater relevance to visual feedback, which becomes important for avoiding obstacles, navigation and informing future actions. Conversely, we found a locomotion-induced 'network switch' in RSP, wherein opposing sub-networks were unmasked by different behavioral states, potentially mediated by a population of locomotion-suppressed FS interneurons. During locomotion, highly locally coupled units were suppressed, and cells became less locally affiliated and more diversely coupled to distal cortical areas, including the visual cortex. This gating of information from sensory areas may reflect RSP's role in navigation, as it is thought to integrate sensory and contextual cues with the animal's internal model of the environment ${ }^{28}$.

We hypothesize that the effect of locomotion on affiliations in V1 and RSP might reflect a sensory-feedback processing mode ${ }^{31}$, whereby both V1 and RSP units are more strongly influenced by sensory input when navigating. If locomotion gates inputs to RSP from other regions, the locomotion-suppressed putative FS inter-neurons might participate in this gating, perhaps acting to shunt long-range sensory inputs during quiescence. This could serve as a circuit mechanism for binding activity within the DMN in passive subjects. V1 and RSP also have many connections with subcortical areas, and during navigation these areas may interact more robustly with RSP neurons. Indeed, we found evidence that SC neurons are more correlated with V1 and RSP activity during locomotion than during quiescence, perhaps to dynamically modulate orienting and other innate motor programs during bouts of locomotion or navigation. RSP is anatomically situated to act as an interface between ego-centric and allocentric representations of an animal's environment ${ }^{28}$, serving to update the animal's internal representation with external sensory cues. We propose a model whereby a strongly locally correlated RSP network is silenced during locomotion, unmasking the activity of cells with stronger affiliations with distal sensory and motor areas, perhaps reflecting a shift in RSP processing whereby the allocentric representations of the environment (for example, head direction) are dynamically updated by sensory feedback from other cortical areas in order to reduce error accumulation during locomotion ${ }^{30}$ (Fig. 5).

\section{Methodological considerations}

In this study, we developed an approach for mapping long-range affiliations of individual neurons in behaving mice. Neurons' unique, heterogeneous and reproducible affiliation patterns suggest that even neighboring neurons participate in different long-range subnetworks. These maps are correlative, and we cannot determine whether these affiliations represent a direct or shared latent influence with other brain regions. Furthermore, due to the complexity of intracortical projections and cortico-thalamo-cortical pathways, and given the temporal smearing inherent in ongoing calcium signals, we cannot reliably infer directed 
relationships using this approach. However, we were able to identify distinct motifs of affiliation for RSP and V1 neurons that could be remapped reliably depending on the animal's behavioral state. While VIP neurons are known to mediate the disinhibitory effect of locomotion on neural dynamics in $\mathrm{V} 1^{38}$, we have identified a sub-population of FS interneurons that may be involved in mediating a similar effect in RSP. Future recordings in a number of molecularly identified neural populations in RSP of locomoting animals are required to identify circuit mechanisms underlying locomotion-dependent remapping of RSP activity. Finally, further work may employ this method to map the dynamics of distributed neuronal affiliations during complex behaviors and shed light on how task-relevant information is dynamically routed to shape computations at cellular resolution.

\section{Methods}

All experimental procedures were carried out in accordance with institutional animal welfare guidelines and licensed by the Swiss cantonal veterinary office. TRE-GCaMP6s mice ${ }^{50}$ (Jackson laboratories, https://www.jax.org/strain/024742) were crossed with B6.CBA$\mathrm{Tg}$ (Camk2a-tTA)1Mmay/DboJ mice (Jackson laboratories, JAX 007004) to drive the expression of GCaMP6s in CamKII ${ }^{+}$pyramidal neurons, and B6 PV-Cre mice (JAX 008069) were crossed with Ai14 mice (JAX 007914) to express tdTomato in $\mathrm{PV}^{+}$cells. Animals were housed in a facility using a reversed light cycle, and recordings were taken during their active period. Recordings were made in 11 female and 14 male mice, ranging between P45 and P70. Additional information can be found in the Life Sciences Reporting Summary.

\section{Surgery}

Two to three weeks before recording, mice were prepared for widefield imaging. Animals were anesthetized with a mixture of fentanyl $(0.05 \mathrm{mg}$ per $\mathrm{kg})$, midazolam $(5.0 \mathrm{mg}$ per $\mathrm{kg}$ ) and medetomidine $(0.5 \mathrm{mg}$ per $\mathrm{kg}$ ). The animal's scalp was resected and a head plate was secured to the skull. Four stereotaxically placed marks were made to enable alignment of the imaged brain with the Allen Brain Atlas (http://mouse.brain-map.org/static/atlas) post hoc, using the Allen Brain API (brain-map.org/api/index.html). The exposed skull was cleaned and covered with transparent dental cement to avoid infection and to cover the cut scalp edges (C\&B Metabond). This was polished to enhance the transparency of the preparation. A custom-made three-dimensional (3D) printed light shield was cemented to the skull and head plate.

\section{Electrophysiological recordings}

After recovery, mice were acclimatized to head fixation for a minimum of 2 days. The day before recording, mice were anesthetized with isofluorane, and a small craniotomy was opened over V1, RSP or both. These were kept damp with Ringer's solution and sealed with KwikSil (World Precision Instruments). Recordings were taken on the next day to avoid any residual effects of anesthesia.

On the recording day, animals were head-fixed under a custom-built widefield microscope, the skull and cortex were cleaned with Ringer's solution, and the KwikSil plug was removed from the craniotomy. A custom-designed silicon probe (64 channels, two shanks, 
Neuronexus) was inserted through the dura after being dipped in diI to allow recovery of the probe tracks (1\% solution in ethanol, Sigma-Aldrich). The probes' sites were configured in a tetrode-like fashion for better single unit isolation. The probe consisted of two shanks with 64 sites total, organized into 16 'tetrodes', each consisting of four sites located $25 \mu \mathrm{m}$ apart from each other within-tetrode, and tetrodes spaced $130 \mu \mathrm{m}$ apart from each other. The probe was inserted at an angle of $\sim 45$ degrees from normal of cortex. A small amount of KwikSil or agar was used to cover the exposed cortex after the probe was in place. After allowing the cortex to settle for 20-30 min, recordings were taken using the OpenEphys recording system ${ }^{51}$. Stable recordings lasted between 30 and $100 \mathrm{~min}$. Behavioral and stimulation data, including pulses representing each camera frame, were recorded using OpenEphys, enabling the alignment of electrophysiological with imaging and behavioral data.

Ephys recordings were filtered between 700 and $7000 \mathrm{~Hz}$, and spikes were detected using the Klustakwik suite ${ }^{52}$. Clusters were assigned to individual units by manual inspection, excluding any units without a clear refractory period. Units of amplitude less than $40 \mu \mathrm{V}$, with fewer than 150 spikes total, a rate of refractory violations greater than $5 \%$, or an isolation difference less than 80 were discarded from further analysis ${ }^{53}$ (see Life Sciences Reporting Summary). Units with few spikes were excluded because they would not afford the power necessary to determine correlations with other activity traces. The detected spikes were then binned to match the imaging frames. Units were separated into fast and broad spiking units by their peak-to-trough time using a cutoff of $0.66 \mathrm{~ms}^{8,54}$. For LFP analyses, data were filtered between 0.1 and $150 \mathrm{~Hz}$. The frequency range of $1-4 \mathrm{~Hz}$ was taken for the delta band, $4-8 \mathrm{~Hz}$ for theta and $8-12 \mathrm{~Hz}$ for alpha.

\section{Widefield imaging}

Widefield imaging was performed through the intact skull using a custom-built scope. Data were recorded using a CMOS camera (Pco.edge 5.5, PCO, Germany) with its native software (Camware, PCO). Sixteen-bit images were acquired at a rate of $40 \mathrm{~Hz}$ using global shutter mode. A constant illumination of $470 \mathrm{~nm}$ was provided. In cases where we performed a hemodynamic correction, every third frame was imaged at $405 \mathrm{~nm}$ to track the hemodynamic response. The imaging site was shielded from light contamination using a 3Dprinted blackout barrier glued to the animal's skull. A recording chamber attached to the camera lens locked snugly with this headpiece, and the chamber was secured with KwikSil.

\section{Two-photon imaging}

Four PV-Cre $\times$ tdTomato transgenic mice were injected with GCaMP6f in dorsal RSP, and an imaging window and headplate were fixed on the skull with dental cement. After allowing 2-3 weeks for virus expression, the animals were trained to navigate a virtual reality environment while headfixed on a wheel using custom Labview software on a custom-built $2 p$ scope $^{22}$. After several days of training, calcium activity was imaged at 900 $\mathrm{nm}$ for $40-60 \mathrm{~min}$. The same fields were imaged at $1030 \mathrm{~nm}$ to determine the identity of PV ${ }^{+}$cells. ROIs were drawn by hand using custom software written in MATLAB, and fluorescence traces were converted to $\triangle F F$. Neurons were classified as locomotionsuppressed if their mean fluorescence during epochs of locomotion was significantly lower 
than during epochs of quiescence, using Student's two-sided $t$-test. We also imaged in L2/3 of 2 GCaMP6s-CamKII ${ }^{+}$transgenic mice to determine the correlation of activity in somas with that of the surrounding neuropil in order to estimate possible contributions to the widefield signal.

\section{Histology}

After recordings, animals were deeply anesthetized by intraperitoneal injection of sodium pentobarbital (Esconarkon), transcardially perfused using $0.9 \% \mathrm{NaCl}$ and fixed with $4 \%$ paraformaldehyde (PFA) in $0.1 \mathrm{M}$ phosphate buffer (PB), pH 7.4. Brains were post-fixed overnight in 4\% paraformaldehyde (PFA), washed with $0.1 \mathrm{M} \mathrm{PB}$ and embedded in $4 \%$ agarose (A9539; Sigma), and slices were cut at a thickness of 100-150 $\mu \mathrm{m}$ using a vibratome (Hyrax V50 Microtome). Slices were counterstained with DAPI ( $2.5 \mu \mathrm{g} \mathrm{ml}^{-1}$ in phosphate buffered saline (PBS) $+0.1 \%$ Triton) and then mounted with a hard-set mounting medium (2.5\% DABCO (D27802; Sigma), 10\% polyvinyl alcohol (P8136; Sigma), 5\% glycerol, $25 \mathrm{mM}$ Tris buffer $\mathrm{pH}$ 8.4). Images were acquired with the Zeiss Axio Scan Slide Scanner (Axio Scan.Z1, Zeiss) using a 10x objective. DiI tracks were used to confirm probe placement and to estimate recording depth. In making depth estimates, tissue was assumed to have shrunk by $30 \%$, as is commonly reported for fixed brain tissue.

\section{Behavioral recordings}

Awake animals were head fixed under the microscope and free to run on a styrofoam wheel. For most recordings, short movies taken from nature documentaries were presented to the eye contralateral to the recording site. Movies were presented using Psych toolbox ${ }^{55}$ and synchronized with the electrophysiological and imaging recordings. A subset of recordings were taken with the animals in the dark or running through a virtual reality corridor yoked to the velocity of their running wheel.

Epochs of locomotion were defined as times when the animal's locomotion persisted above $1 \mathrm{~cm}$ per $\mathrm{s}$ for more than $2 \mathrm{~s}$, the same threshold used in previous studies ${ }^{21}$.

\section{Data analysis}

Raw imaging data were spatially binned $3 \times 3$, loaded into MATLAB as a mapped tensor ${ }^{56}$ and converted to $\triangle F F$, by subtracting a moving baseline from the fluorescence at a given time point, then dividing by the same baseline value. The moving baseline value was calculated as the fifth percentile of points from the preceding $20 \mathrm{~s}$ of data. We undertook to control for possible confounding effects of the hemodynamic signal on SpAMs. We imaged from three animals, interleaving imaging at $470 \mathrm{~nm}$ and at $405 \mathrm{~nm}$ on every third frame, which allowed us to correct for the hemodynamic component of the signal. We interpolated the hemodynamic and calcium traces to correspond in time and then divided the calcium signal by the hemodynamic signal to create the hemodynamic-corrected signal. We calculated $\triangle F F$ for both the corrected and uncorrected traces (Supplementary Fig. 4). A map of the correlation of the hemodynamic-corrected and uncorrected $\Delta F F$ trace over the brain, averaged over three animals, showed no systematic difference across the imaged brain surface (Supplementary Fig. 4a). For each of our three animals, we then deconvolved the $\Delta F F$ trace of a pixel over RSP to approximate spiking activity using the nerds deconvolution 
toolbox57 (Supplementary Fig. 4b-d; https://github.com/KordingLab/nerds/). We generated 60 simulated spike trains from these data by randomly removing spikes from this deconvolved signal, and used these spike trains to calculate SpAMs for the uncorrected and corrected movies. SpaMs were similar in both cases, and there was no systematic change in maps between epochs of locomotion and quiescence, suggesting that it is indeed a property of the correlational structure of individual cells, and not hemodynamic patterns, that accounts for the remapping we found in RSP. Other groups similarly found that hemodynamic and flavoprotein signals contribute minimally compared with the calcium responses ${ }^{12,58}$.

To build SpAMs, spike trains were binned to match imaging frames, and SpAMs were calculated by taking the partial correlation of each unit's spike train with each pixel's $\Delta F F$ with respect to global brain fluorescence (mean $\Delta F F$ for all pixels covering cortex). $P$ values for these maps were Benjamini-Hochberg corrected for multiple comparisons, with a significance threshold set at a 5\% false positive rate. Pixels not significantly different from zero are transparent in the presented maps. Significance was also confirmed by comparison with SpAMs from shuffled spike trains. Spike trains were split into $1 \mathrm{~s}$ intervals (to maintain the short-term structure of spike correlations), randomly rearranged, and used to calculate spike-triggered activity maps of calcium signal (SpAMs) (Fig. 1g, gray bars). Finally, to test for reproducibility of these maps, data were split into interleaved epochs. SpAMs were calculated for these divided data, and their pixel-by-pixel correlation is presented in Supplementary Fig. 5. Units that did not have stable SpAMs were excluded from the study ( $N=6$ units, with SpAM self-correlation less than 0.2).

The correlation values in SpAMs are low. First, calcium imaging is slow compared with neural spiking, and this temporal disparity will lead to lower correlations. Second, each imaged pixel represents the summed spiking activity of many neuronal somata as well as contributions from axons and dendrites. To address the first point, we wished to determine the maximum correlation we could hope to find between a discrete spike train and fluorescence data. To correct for the disparity in the temporal course of these data, we could either deconvolve the imaging data or convolve the spiking with a decaying exponential fit on the fluorescence traces. We decided to perform convolution on the spiking data, as the imaging signal probably reflects spiking and subthreshold signals, which cannot be deconvolved into discrete events. We summed all single and multiunit activity to approximate a population activity trace and convolved it with a decaying exponential fit to simulate a calcium trace. The correlation between the convolved multiunit activity and the unconvolved activity was, on average, $0.3 \pm 0.1$ ( $N=14$ mice). This represents the highest correlation we could hope to see between the spiking and imaging data, without noise and non-spike-related signals in the fluorescence. We also recalculated all SpAMs using convolved spike trains. This did not change the spatial correlation patterns in any appreciable way (Supplementary Fig. 3a,b), but correlation values were on average five times higher.

We also wished to determine whether variations in our maps were only a result of shared latent input from running signals, so we recalculated all SpaMs using a partial correlation between spiking and fluorescence with respect to the running signal. SpaMs remained 
extremely similar between these maps and those calculated elsewhere in the paper (Supplementary Fig. 3c).

Population coupling was calculated for each unit as described previously ${ }^{8}$. Each unit's coupling was $z$-scored in order to compare across subjects. Firing rate modulation by locomotion was taken as the average spike rate during locomotion divided by the average spike rate during quiescence (units that did not spike in either of these conditions were excluded from the analyses).

To calculate spike train correlations, data were binned to $24 \mathrm{~ms}$ (Fig. 4b). Correlations dropped during locomotion for both V1 and RSP units. In RSP, this could not be accounted for by the reduction in firing rate, which we tested by randomly omitting spikes from a unit's spike train in accordance with the average rate reduction and then calculating correlations for these rate-corrected spike trains (Fig. 4b, paired $t$-test for quiescence (Q) vs. locomotion (L), two-sample $t$-test for 1,000 repetitions of the firing rate-resampled correlations (Q') vs. Q and L).

\section{Data and code availability}

The data and code used in this study are available from the corresponding author upon reasonable request.

\section{Statistics}

Sample sizes were not statistically determined but were consistent with previous papers using related methodology ${ }^{12}$. Data distribution was assumed to be normal, but this was not formally tested. Data collection and analysis were not performed blind to the conditions of the experiments. Data collection was not randomized, but visual stimulation was randomized - a small set of movies were shown in random order, at random intervals, to which the experimenter was blind.

All reported correlations are Pearson correlations unless otherwise stated. Each pixel of a SpAM represents the Pearson correlation of the unit's recorded spiking with the activity in that pixel. The significance of each pixel was determined using Benjamini-Hochberg correction on the $P$ values of all pixel correlations, with the significance threshold set at a $5 \%$ false positive rate.

Two-dimensional correlations between two matrices, A and B, are calculated as follows:

$$
r=\frac{\sum_{m} \sum_{n}\left(A_{m n}-\bar{A}\right)\left(B_{m n}-\bar{B}\right)}{\sqrt{\left(\sum_{m} \sum_{n}\left(A_{m n}-\bar{A}\right)^{2}\right)\left(\sum_{m} \sum_{n}\left(B_{m n}-\bar{B}\right)^{2}\right)}}
$$

where $\bar{A}$ and $\bar{B}$ are the mean of matrices $\mathrm{A}$ and $\mathrm{B}$, respectively, and $m$ and $n$ denote matrix rows and columns.

The significance of differences between group means was determined using Student's $t$-test, with Bonferroni correction for multiple comparisons where applicable. 
For the cluster analysis of RSP unit SpAMs during locomotion versus quiescence, we performed K-means clustering and determined the optimal number of clusters using the Calinski-Harabasz index for internal validation. We used the Kolmogorov-Smirnov test to determine whether the number of clusters for these two conditions was probably drawn from different distributions.

\section{Contributions to the widefield signal}

The correlation between the local fluorescence trace and the multiunit spiking activity convolved with a decaying exponential fit on the imaging data was, on average, $0.6 \pm 0.1$ for $\mathrm{V} 1$ and $0.6 \pm 0.1$ for RSP, in agreement with previously published values ${ }^{59}$. We undertook to distinguish various contributions to the fluorescence signals by fitting the local fluorescence with a generalized linear model (GLM) whose predictors were instantaneous spiking, velocity (smoothed over 0.5 seconds) and LFP power (Supplementary Fig. 7). Spiking signals explained $\sim 50 \%$ of the fluorescence signal, running $\sim 10 \%$ and LFP $\sim 3 \%$.

The widefield fluorescence signal is probably a weighted sum of predominantly local dendrites, axons and cell bodies, with a small contribution from distal axons. We expect the majority of the local neuropil signal to follow local spiking. Indeed, using two-photon imaging in the same GCaMP6s-CamKII mice used in the rest of this study, we found that the average correlation of L2/3 soma with surrounding neuropil (largely cross sections of apical dendrites) was $0.9 \pm 0.1$ ( $N=516$ cells, two mice, four recording sessions). We expect passing superficial axons to make only a minor contribution to this signal: a previous study blocked local glutamatergic signaling and found that between $65 \%$ and $90 \%$ of the widefield calcium responses were abolished in Thy 1 mice, suggesting that fluorescence signals largely reflect local activity and not long-range inputs ${ }^{36}$.

\section{Reporting Summary}

Further information on research design is available in the Nature Research Reporting Summary linked to this article.

\section{Supplementary Material}

Refer to Web version on PubMed Central for supplementary material.

\section{Acknowledgements}

The authors thank A. Garner and G. Keller for help with two-photon imaging in RSP, L. Hoermann for performing surgeries and histology for this project, and S. Hofer, P. Znamenskiy and A. Naka for feedback and discussions on this project and comments on the manuscript. This work was supported by the Swiss National Science Foundation (SNSF 31003A_169802 to T.D.M.-F.), the Wellcome Trust (090843/E/09/Z core grant to the Sainsbury Wellcome Centre), the EMBO Long-term Fellowship (ALTF 1481-2014 to K.B.C.), the HFSP Postdoctoral Fellowship (LT000414/2015-L to K.B.C.) and the Branco Weiss-Society in Science grant (K.B.C.).

\section{References}

1. Harris KD, Mrsic-Flogel TD. Cortical connectivity and sensory coding. Nature. 2013; 503:51-58. [PubMed: 24201278]

2. Ko H, et al. Functional specificity of local synaptic connections in neocortical networks. Nature. 2011; 473:87-91. [PubMed: 21478872] 
3. Cossell L, et al. Functional organization of excitatory synaptic strength in primary visual cortex. Nature. 2015; 518:399-403. [PubMed: 25652823]

4. Holmgren C, Harkany T, Svennenfors B, Zilberter Y. Pyramidal cell communication within local networks in layer 2/3 of rat neocortex. J Physiol (Lond). 2003; 551:139-153. [PubMed: 12813147]

5. Yoshimura Y, Callaway EM. Fine-scale specificity of cortical networks depends on inhibitory cell type and connectivity. Nat Neurosci. 2005; 8:1552-1559. [PubMed: 16222228]

6. Morgenstern NA, Bourg J, Petreanu L. Multilaminar networks of cortical neurons integrate common inputs from sensory thalamus. Nat Neurosci. 2016; 19:1034-1040. [PubMed: 27376765]

7. Gentet LJ, Avermann M, Matyas F, Staiger JF, Petersen CCH. Membrane potential dynamics of GABAergic neurons in the barrel cortex of behaving mice. Neuron. 2010; 65:422-435. [PubMed: 20159454]

8. Okun M, et al. Diverse coupling of neurons to populations in sensory cortex. Nature. 2015; 521:511-515. [PubMed: 25849776]

9. Markov NT, et al. A weighted and directed interareal connectivity matrix for macaque cerebral cortex. Cereb Cortex. 2014; 24:17-36. [PubMed: 23010748]

10. Zingg B, et al. Neural networks of the mouse neocortex. Cell. 2014; 156:1096-1111. [PubMed: 24581503]

11. Oh SW, et al. A mesoscale connectome of the mouse brain. Nature. 2014; 508:207-214. [PubMed: 24695228]

12. Xiao D, et al. Mapping cortical mesoscopic networks of single spiking cortical or sub-cortical neurons. eLife. 2017; 6:6.

13. Arieli A, Sterkin A, Grinvald A, Aertsen A. Dynamics of ongoing activity: explanation of the large variability in evoked cortical responses. Science. 1996; 273:1868-1871. [PubMed: 8791593]

14. Tsodyks M, Kenet T, Grinvald A, Arieli A. Linking spontaneous activity of single cortical neurons and the underlying functional architecture. Science. 1999; 286:1943-1946. [PubMed: 10583955]

15. Barson D, et al. Simultaneous mesoscopic and two-photon imaging of neuronal activity in cortical circuits. bioRxiv. 2018; doi: 10.1101/468348

16. Hofer SB, et al. Differential connectivity and response dynamics of excitatory and inhibitory neurons in visual cortex. Nat Neurosci. 2011; 14:1045-1052. [PubMed: 21765421]

17. Bock DD, et al. Network anatomy and in vivo physiology of visual cortical neurons. Nature. 2011; 471:177-182. [PubMed: 21390124]

18. Kerlin AM, Andermann ML, Berezovskii VK, Reid RC. Broadly tuned response properties of diverse inhibitory neuron subtypes in mouse visual cortex. Neuron. 2010; 67:858-871. [PubMed: 20826316]

19. Poulet JFA, Petersen CCH. Internal brain state regulates membrane potential synchrony in barrel cortex of behaving mice. Nature. 2008; 454:881-885. [PubMed: 18633351]

20. Otazu GH, Tai L-H, Yang Y, Zador AM. Engaging in an auditory task suppresses responses in auditory cortex. Nat Neurosci. 2009; 12:646-654. [PubMed: 19363491]

21. Niell CM, Stryker MP. Modulation of visual responses by behavioral state in mouse visual cortex. Neuron. 2010; 65:472-479. [PubMed: 20188652]

22. Keller GB, Bonhoeffer T, Hübener M. Sensorimotor mismatch signals in primary visual cortex of the behaving mouse. Neuron. 2012; 74:809-815. [PubMed: 22681686]

23. Schneider DM, Nelson A, Mooney R. A synaptic and circuit basis for corollary discharge in the auditory cortex. Nature. 2014; 513:189-194. [PubMed: 25162524]

24. Vinck M, Batista-Brito R, Knoblich U, Cardin JA. Arousal and locomotion make distinct contributions to cortical activity patterns and visual encoding. Neuron. 2015; 86:740-754. [PubMed: 25892300]

25. McGinley MJ, et al. Waking state: rapid variations modulate neural and behavioral responses. Neuron. 2015; 87:1143-1161. [PubMed: 26402600]

26. Raichle ME. The brain's default mode network. Annu Rev Neurosci. 2015; 38:433-447. [PubMed: 25938726]

27. Hillman EMC. Coupling mechanism and significance of the BOLD signal: a status report. Annu Rev Neurosci. 2014; 37:161-181. [PubMed: 25032494] 
28. Alexander AS, Nitz DA. Retrosplenial cortex maps the conjunction of internal and external spaces. Nat Neurosci. 2015; 18:1143-1151. [PubMed: 26147532]

29. Vann SD, Aggleton JP, Maguire EA. What does the retrosplenial cortex do? Nat Rev Neurosci. 2009; 10:792-802. [PubMed: 19812579]

30. Bicanski A, Burgess N. Environmental anchoring of head direction in a computational model of retrosplenial cortex. J Neurosci. 2016; 36:11601-11618. [PubMed: 27852770]

31. Dipoppa M, et al. Vision and locomotion shape the interactions between neuron types in mouse visual cortex. Neuron. 2018; 98:602-615.e8. [PubMed: 29656873]

32. Wang Q, Burkhalter A. Area map of mouse visual cortex. J Comp Neurol. 2007; 502:339-357. [PubMed: 17366604]

33. Yamawaki N, Radulovic J, Shepherd GMG. A corticocortical circuit directly links retrosplenial cortex to M2 in the mouse. J Neurosci. 2016; 36:9365-9374. [PubMed: 27605612]

34. Shibata H, Kondo S, Naito J. Organization of retrosplenial cortical projections to the anterior cingulate, motor, and prefrontal cortices in the rat. Neurosci Res. 2004; 49:1-11. [PubMed: 15099698]

35. Stafford JM, et al. Large-scale topology and the default mode network in the mouse connectome. Proc Natl Acad Sci USA. 2014; 111:18745-18750. [PubMed: 25512496]

36. Makino H, et al. Transformation of cortex-wide emergent properties during motor learning. Neuron. 2017; 94:880-890.e8. [PubMed: 28521138]

37. Liu Y-J, et al. Tracing inputs to inhibitory or excitatory neurons of mouse and cat visual cortex with a targeted rabies virus. Curr Biol. 2013; 23:1746-1755. [PubMed: 23993841]

38. Fu Y, et al. A cortical circuit for gain control by behavioral state. Cell. 2014; 156:1139-1152. [PubMed: 24630718]

39. Erisken S, et al. Effects of locomotion extend throughout the mouse early visual system. Curr Biol. 2014; 24:2899-2907. [PubMed: 25484299]

40. Pakan JM, et al. Behavioral-state modulation of inhibition is context-dependent and cell type specific in mouse visual cortex. eLife. 2016; 5:e14985. [PubMed: 27552056]

41. Dadarlat MC, Stryker MP. Locomotion enhances neural encoding of visual stimuli in mouse V1. J Neurosci. 2017; 37:3764-3775. [PubMed: 28264980]

42. King AJ. The superior colliculus. Curr Biol. 2004; 14:R335-R338. [PubMed: 15120083]

43. Zhang S, et al. Selective attention. Long-range and local circuits for top-down modulation of visual cortex processing. Science. 2014; 345:660-665. [PubMed: 25104383]

44. Makino H, Komiyama T. Learning enhances the relative impact of top-down processing in the visual cortex. Nat Neurosci. 2015; 18:1116-1122. [PubMed: 26167904]

45. Fiser A, et al. Experience-dependent spatial expectations in mouse visual cortex. Nat Neurosci. 2016; 19:1658-1664. [PubMed: 27618309]

46. Leinweber M, Ward DR, Sobczak JM, Attinger A, Keller GB. A sensorimotor circuit in mouse cortex for visual flow predictions. Neuron. 2017; 95:1420-1432.e5. [PubMed: 28910624]

47. Manita S, et al. A top-down cortical circuit for accurate sensory perception. Neuron. 2015; 86:1304-1316. [PubMed: 26004915]

48. Renart A, Machens CK. Variability in neural activity and behavior. Curr Opin Neurobiol. 2014; 25:211-220. [PubMed: 24632334]

49. Schölvinck ML, Saleem AB, Benucci A, Harris KD, Carandini M. Cortical state determines global variability and correlations in visual cortex. J Neurosci. 2015; 35:170-178. [PubMed: 25568112]

50. Wekselblatt JB, Flister ED, Piscopo DM, Niell CM. Large-scale imaging of cortical dynamics during sensory perception and behavior. J Neurophysiol. 2016; 115:2852-2866. [PubMed: 26912600]

51. Siegle JH, et al. Open Ephys: an open-source, plugin-based platform for multichannel electrophysiology. J Neural Eng. 2017; 14

52. Kadir SN, Goodman DFM, Harris KD. High-dimensional cluster analysis with the masked EM algorithm. Neural Comput. 2014; 26:2379-2394. [PubMed: 25149694]

53. Schmitzer-Torbert N, Jackson J, Henze D, Harris K, Redish AD. Quantitative measures of cluster quality for use in extracellular recordings. Neuroscience. 2005; 131:1-11. [PubMed: 15680687] 
54. Barthó $P$, et al. Characterization of neocortical principal cells and interneurons by network interactions and extracellular features. J Neurophysiol. 2004; 92:600-608. [PubMed: 15056678]

55. Brainard DH. The psychophysics toolbox. Spat Vis. 1997; 10:433-436. [PubMed: 9176952]

56. Muir DR, Kampa B. FocusStack and StimServer: A new open source MATLAB toolchain for visual stimulation and analysis of two-photon calcium neuronal imaging data. Front Neuroinformatics. 2015; 8:85.

57. Dyer, EL; Studer, C; Robinson, JT; Baraniuk, RG. A robust and efficient method to recover neural events from noisy and corrupted data. 6th International IEEE/EMBS Conference on Neural Engineering (NER); 2013. 593-596.

58. Vanni MP, Murphy TH. Mesoscale transcranial spontaneous activity mapping in GCaMP3 transgenic mice reveals extensive reciprocal connections between areas of somatomotor cortex. $\mathrm{J}$ Neurosci. 2014; 34:15931-15946. [PubMed: 25429135]

59. Ma Y, et al. Resting-state hemodynamics are spatiotemporally coupled to synchronized and symmetric neural activity in excitatory neurons. Proc Natl Acad Sci USA. 2016; 113:E8463E8471. [PubMed: 27974609] 

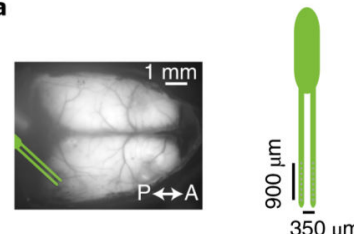

b

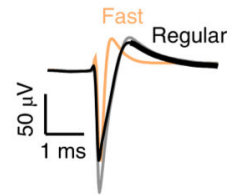

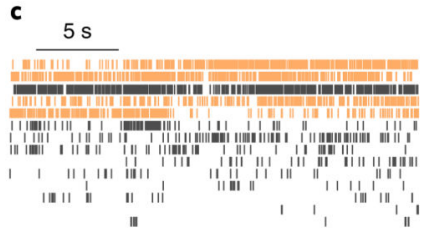

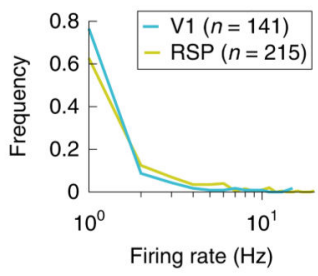

d
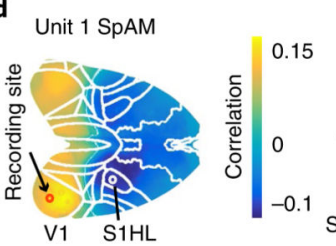

$\stackrel{N}{\sim} \stackrel{ }{\circ} \underset{5 s}{-} \begin{aligned} & -\Delta F / F \\ & - \text { Convolved spike rate }\end{aligned}$
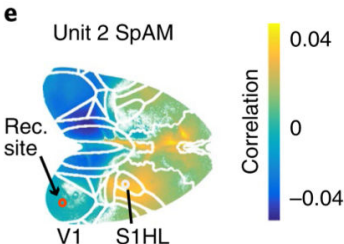

$\stackrel{N}{\sim} \bigsqcup_{5 \mathrm{~s}} \begin{aligned} & -\Delta F / F \\ & - \text { Convolved spike rate }\end{aligned}$

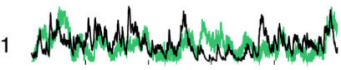

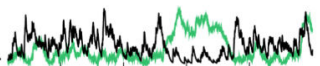
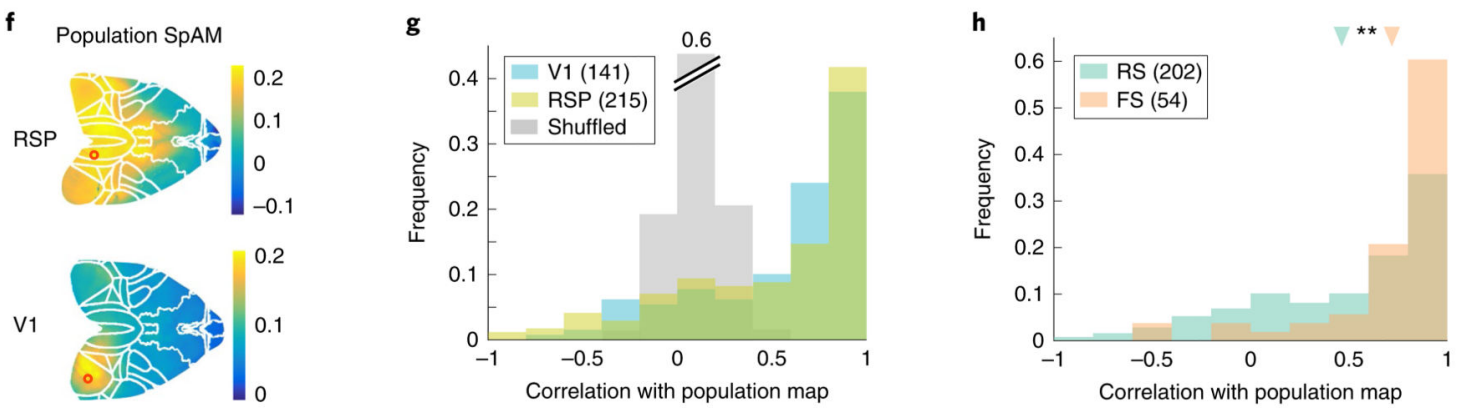

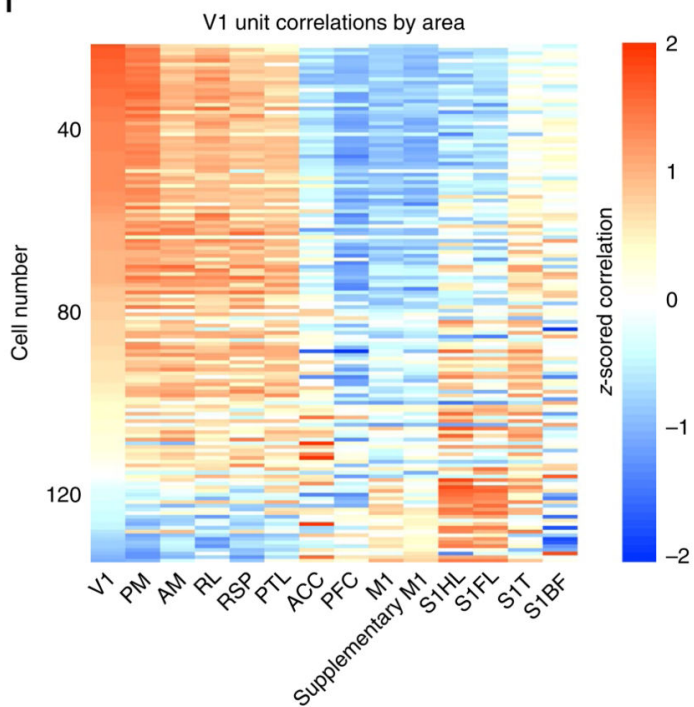

j

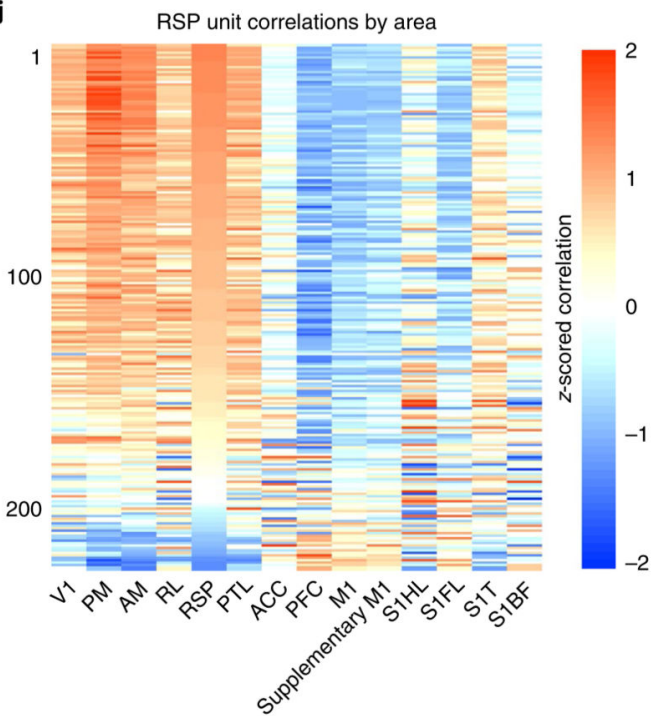

Fig. 1. Neurons exhibit diverse affiliations with global networks.

a, Example imaging field and recording probe design. b. Example waveforms from three recorded units. Fast-spiking units (FS, putative interneurons) could be distinguished from regular-spiking units (RS) by their waveform. c, Example spike trains for 14 units recorded in V1 (left) and histogram of firing rates (right). d, Left: example spike-triggered activity map (SpAM) for unit recorded in V1, registered to the Allen Brain Atlas (outlined) using stereotaxically placed skull landmarks. Right: the unit's spike train was convolved with an exponential decay fit from the calcium data (black), plotted with the fluorescence trace from 
a pixel in V1 (top) and in hindlimb somatosensory cortex (S1HL, bottom). Individual pixels with correlation values not significantly different from zero are transparent (Pearson correlation, $P$ values Benjamini-Hochberg corrected, with significance threshold set at a 5\% false-positive rate). e, Same as $\mathbf{d}$, for a simultaneously recorded V1 unit (Pearson correlation, $P$ values Benjamini-Hochberg corrected, with significance threshold set at a 5\% false-positive rate). f, Example population SpAMs for recordings taken in V1 (top) and RSP (bottom), whereby the summed spiking activity was correlated with activity in each pixel across the cortex. g, Two-dimensional correlation coefficients between individual SpAMs and the population SpAM for recordings in V1 and RSP. Spike trains were randomized to calculate shuffled SpAMs (gray). For V1 units, 73\% of SpAMs were significantly correlated and $11 \%$ were significantly anticorrelated with the population SpAM (calculated as percent of units that fall outside the $95 \%$ confidence interval of shuffled SpAM distribution). In RSP, $65 \%$ and $11 \%$ of neurons were significantly correlated or anticorrelated with the population SpAM, respectively (calculated as percent of units that fall outside the $95 \%$ confidence interval of shuffled SpAM distribution). $\mathbf{h}$, FS units (putative interneurons) had SpAMs significantly more similar to the population SpAMs than RS units (as measured by twodimensional correlation, see Methods; means indicated with arrows, two-sided $t$-test, $P=1 \times$ $10^{-4}, \mathrm{~T}=3.8$, degrees of freedom $\left.(\mathrm{df})=354\right)$. $\mathbf{i}$, Mean Pearson correlations with various cortical areas for all recorded V1 units, sorted by correlation with V1. $\mathbf{j}$, Same as $\mathbf{i}$, for RSP units, sorted by Pearson correlation with RSP. 

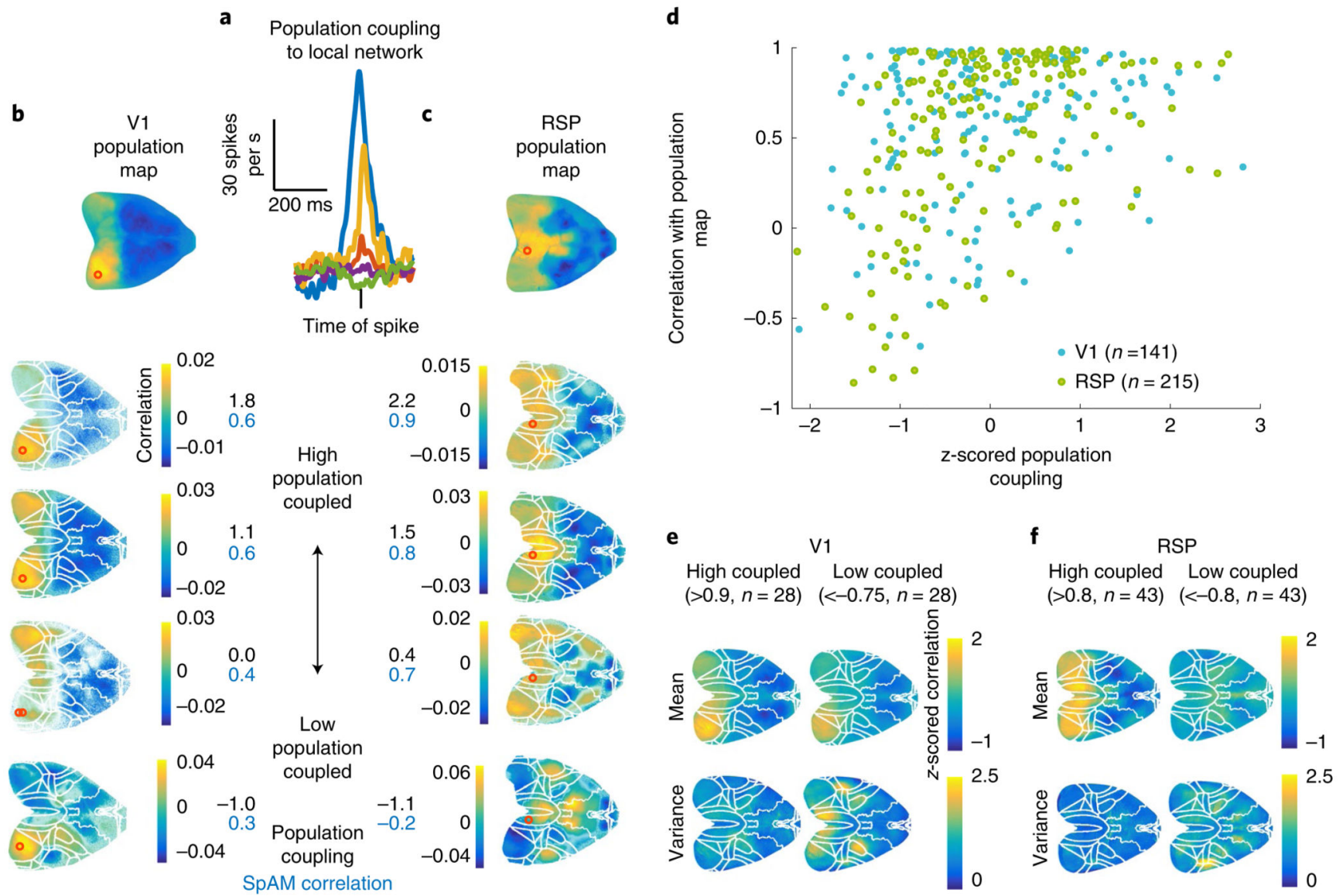

Fig. 2. Units uncoupled to local activity were more probably affiliated with global networks.

a, A unit's population coupling is the correlation between the spiking of a given cell and the sum of spikes from all other simultaneously recorded neurons. Population coupling is shown for five units recorded in V1. b, Example SpAMs for four V1 units (Pearson correlation). Top: population SpAM with recording site circled in red. All SpAMs were aligned to the Allen Brain Atlas (overlaid). A unit's $Z$-scored population coupling is shown in black, and its $2 \mathrm{D}$ correlation with the population SpAM in blue. c, Same as $\mathbf{b}$, for example units recorded in RSP. d, Population coupling versus unit's SpAM 2D correlation with the population SpAM. High-coupled units had SpAMs more similar to the population SpAM, while the SpAM values of low-coupled units were more diverse in both V1 (linear regression, slope $=0.44, R=0.18, P=0.02$ ) and $\mathrm{RSP}$ (linear regression, slope $=0.92, R=$ $0.45, P=1 \times 10^{-9}$ ). e, Mean (top row) and variance (bottom) of SpAMs for the highestcoupled (left) and lowest-coupled (right) V1 units (Pearson correlations). The low-coupled V1 units exhibited common affiliation motifs with RSP, S1HL and M1HL, a lateral parietal area, and barrel S1. SpAMs were $z$-scored before averaging. $\mathbf{f}$, Same as e, for recordings in RSP (Pearson correlations). Common affiliation motifs included secondary motor cortex, S1HL and M1HL, barrel cortex, and a lateral parietal area. 


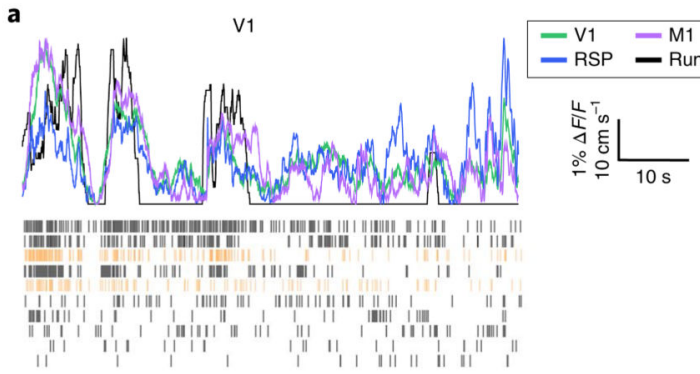

b

V1 average SpAMs

Quiescence Locomotion

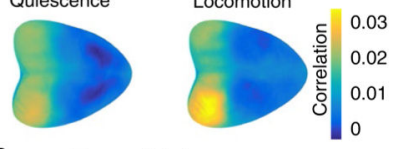

Run modulation SpAM correlation Q vs. L
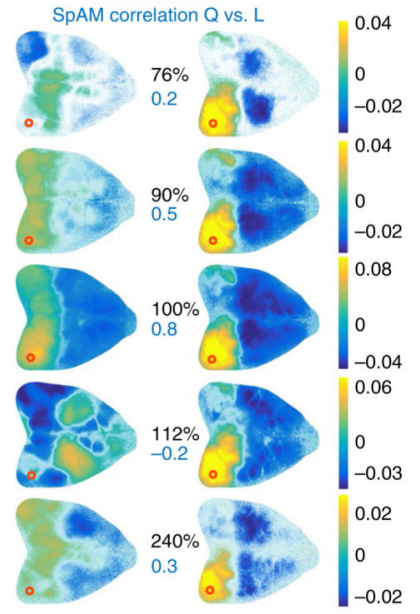

i

Average SpAM locomotion
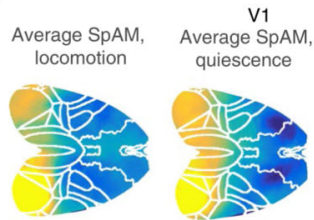

Average SpA

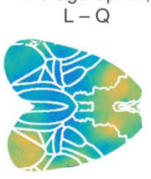

j

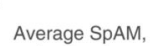

RSP

Average SpAM

locomation
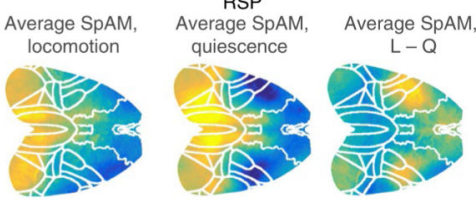

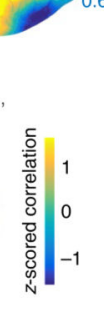

d

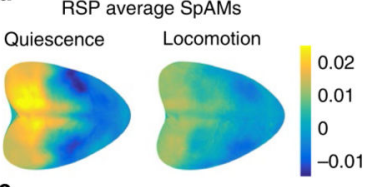

e

Run modulation

SpAM correlation Q vs. L
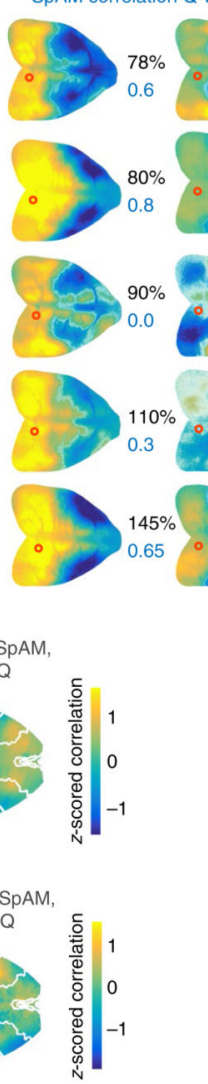
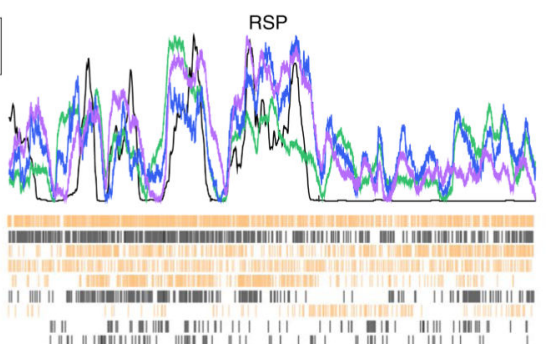

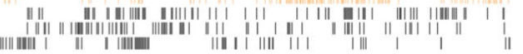
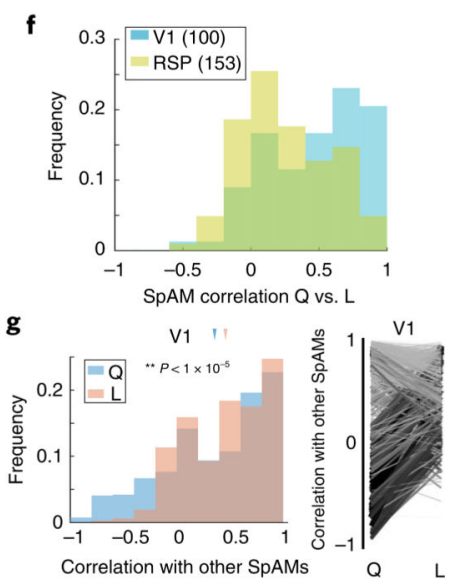

h

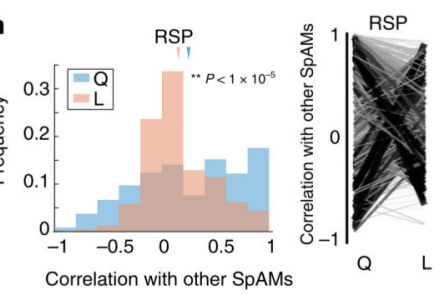

Fig. 3. Global affiliation patterns are state-specific.

a, Fluorescence traces from three areas overlaid with running speed, and spike rasters of RS (black) and FS (orange) units recorded in V1 (left) and RSP (right). b, Average SpAM for units recorded in V1 during quiescence (left) and locomotion (right) (SpAMs calculated using Pearson correlation, $N=100$ units). c, SpAMs (Pearson correlation) for five simultaneously recorded V1 units during quiescence (Q; left) and locomotion (L; right). The effect of running on a unit's change in firing rate is indicated in black (relative to quiescence) and the 2D correlation of its quiescence versus locomotion SpAMs is shown in 
blue. Increases or decreases in firing rate during locomotion were not necessarily associated with higher or lower SpAM correlations. Red circle indicates recording location. d, Same as b, for all RSP units (Pearson correlation, $N=153$ units). e, Same as $\mathbf{c}$ for example RSP units (Pearson correlation). f, Histogram of the 2D correlation between each unit's SpAM during quiescence and during locomotion ( $N=100$ units in V1, $N=153$ units in RSP). RSP SpAMs remapped more extensively than V1 units upon locomotion. Units whose activity was suppressed by more than 50\% during locomotion were excluded to avoid including any noise-dominated maps in these analyses, but including all data did not affect the main trend. g, Pairwise 2D correlations of V1 SpAMs during quiescence and locomotion. V1 SpAMs become more similar to one another during locomotion $(N=100$ units, two-sided paired $t$ test, $P=1 \times 10^{-5}, t=4.4, \mathrm{df}=698$ ). Right: pairwise 2D correlations of V1 SpAMs during quiescence versus locomotion. Lines were weighted by the magnitude of the pair's change between the two conditions ( $N=100$ units; thin, light gray lines: small change; thick, black lines: large change). $\mathbf{h}$, Same as $\mathbf{g}$, for RSP ( $N=153$ units, 2D correlation). RSP units' SpAMs become less similar to one another during locomotion $(N=153$ units, two-sided paired $t$-test, $P=4 \times 10^{-10}, t=6.3, \mathrm{df}=960$ ). i, Average of V1 population SpAMs during locomotion (left), quiescence (middle) and their difference (right, $N=5$ mice, Pearson correlations). $\mathbf{j}$, Average of RSP population SpAMs during locomotion (left), quiescence (middle) and their difference (right, $N=5$ mice, Pearson correlations). k, Average difference between V1 and RSP population SpAMs (Pearson correlations) during locomotion and quiescence for different cortical areas (error bars s.e.m.). Corr., correlation. 
a
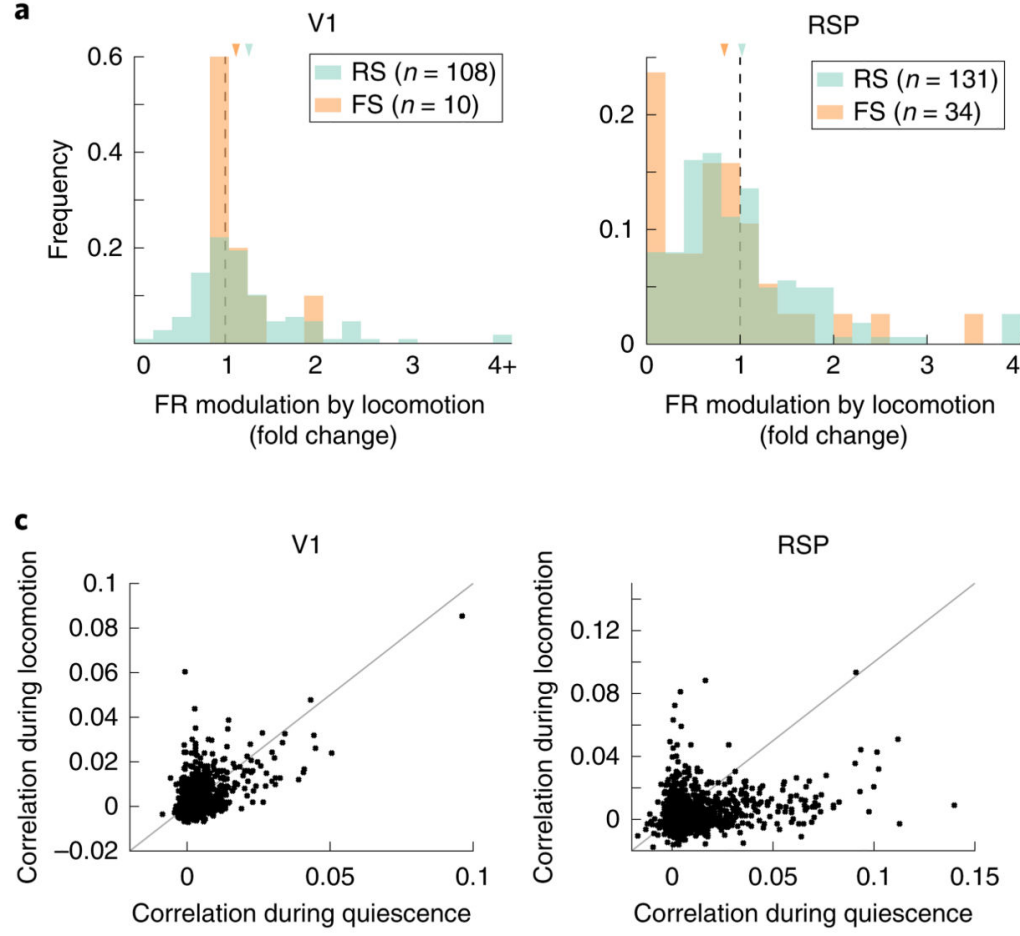

b

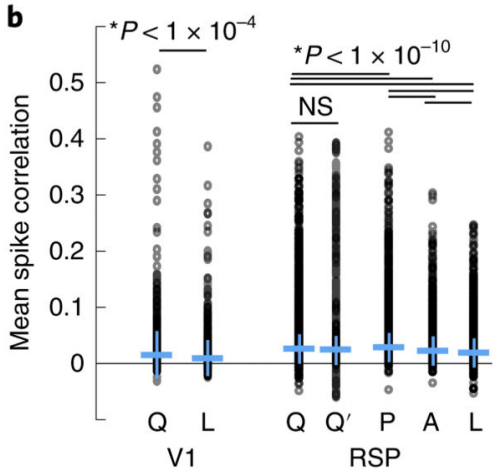

d

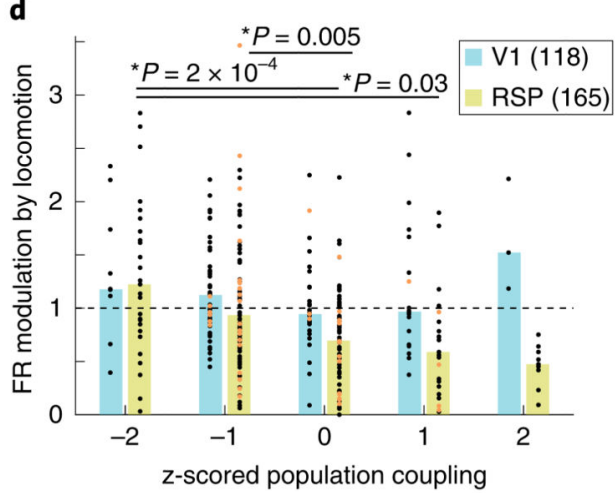

e

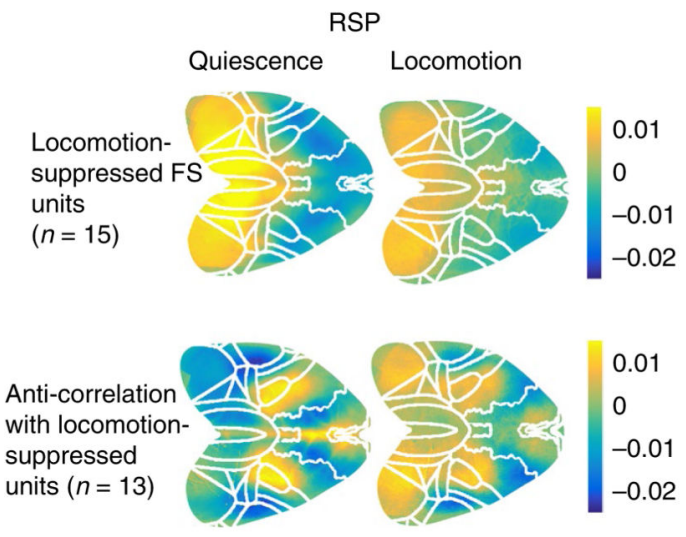

f

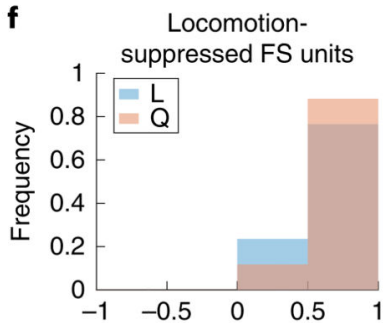

Anti-correlation with locomotion-suppressed FS units

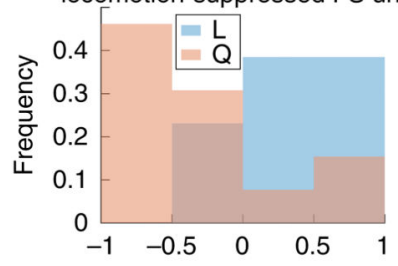

Correlation with population SpAM g

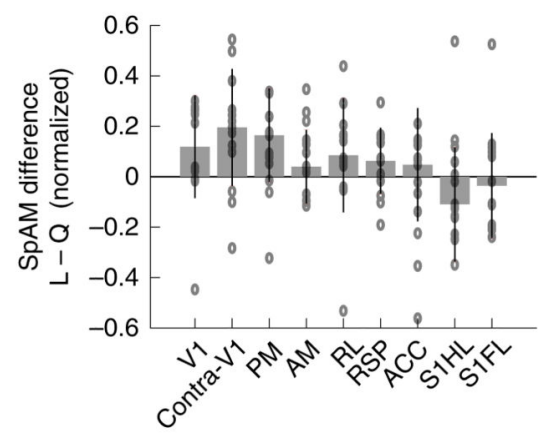

Fig. 4. Locomotion triggers a reorganization of RSP global affiliations.

a, Firing rates of RS and FS neurons increase during locomotion in V1 (left, $N=108 \mathrm{RS}$ units, $N=10 \mathrm{FS}$ units) and decrease for FS units in RSP (right, $N=131 \mathrm{RS}$ units, $N=34 \mathrm{FS}$ units). Many RSP FS units were strongly suppressed by locomotion. Means are indicated with arrows. b, Pairwise spiking Pearson correlations in V1 and RSP drop significantly during locomotion compared with quiescence (pairwise two-sided $t$-test, bars denote standard deviation, $N=1,461 \mathrm{~V} 1$ pairs, $N=4,724$ pairs). This could not be accounted for by the drop in firing rate in RSP units during locomotion (Q', spike-rate corrected Pearson 
correlation; see Methods). Pearson correlations during passive wakefulness (P) were higher than in unmoving animals $(\mathrm{Q})$, and Pearson correlations in aroused animals $(\mathrm{A})$ were significantly lower than in both quiescent and passive animals, but not as low as in locomoting animals (Bonferroni-corrected two-sided $t$-test). c, Spike correlations (Pearson) during quiescence and locomotion for all V1 and RSP units. d, During locomotion, highcoupled RSP units were more probably suppressed (Bonferroni-corrected two-sided $t$-test). The effect of locomotion on V1 units was not related to population coupling. FS units indicated in orange. e, Top row: average SpAMs of locomotion-suppressed FS units in RSP during quiescence and locomotion (Pearson correlation). Bottom row: average SpAMs of RSP units anti-correlated with locomotion-suppressed FS units, during quiescence and locomotion (Pearson correlation). f, Top: 2D correlation with population SpAM for locomotion-suppressed units during quiescence and locomotion ( $N=15$ units). Bottom: 2D correlation with population SpAM for units anti-correlated with locomotion-suppressed units ( $N=13$ units). These SPAMs were anti-correlated with the population SpAM during quiescence but became more similar to it during locomotion. $\mathbf{g}$, Average SpAM difference (locomotion - quiescence, bars denote standard deviation) for the population of cells anticorrelated with locomotion-suppressed FS units ( $N=13$ units). 


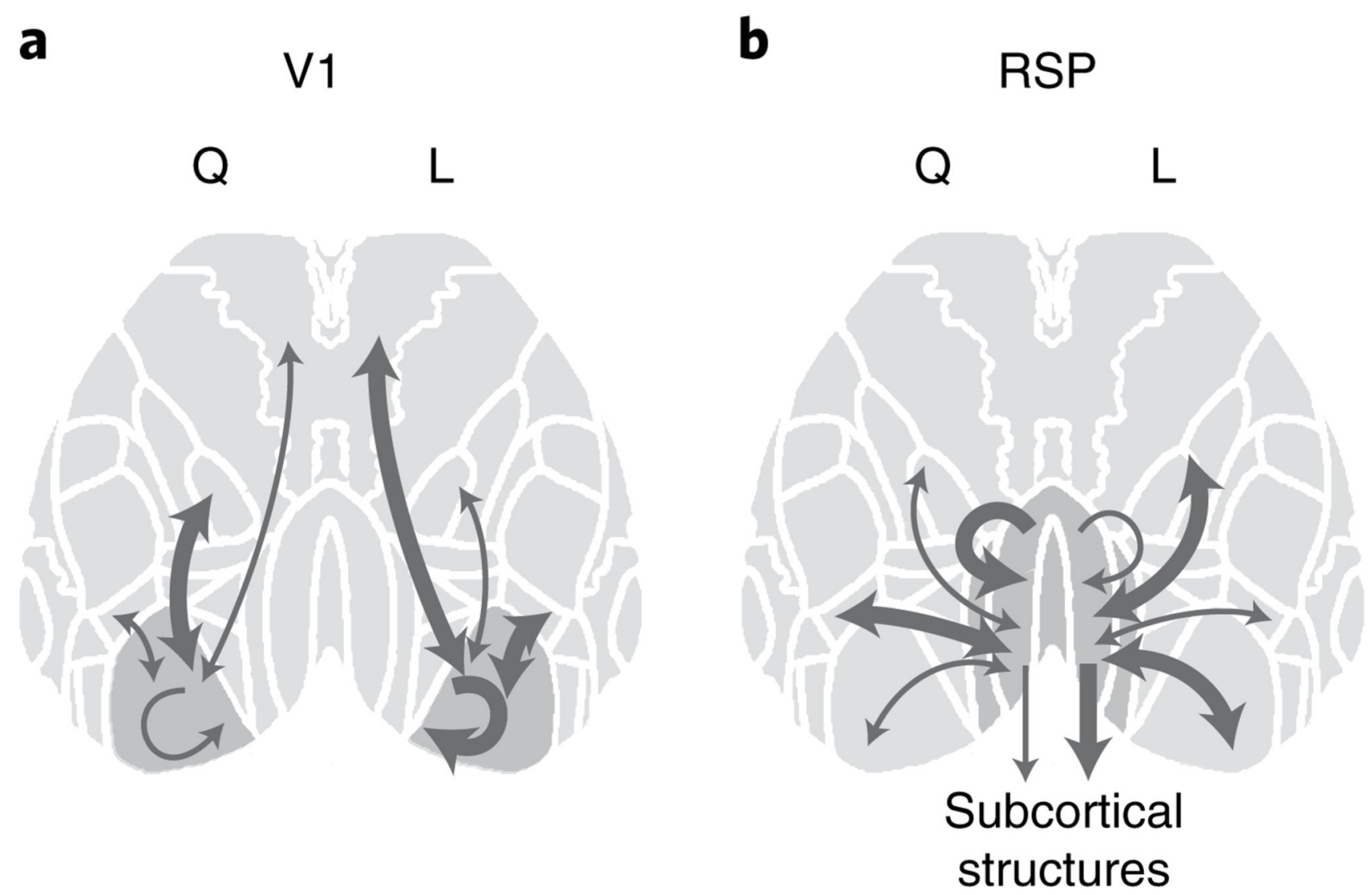

Fig. 5. Schematic of change in affiliation patterns.

a, Schematic of V1 dynamics during quiescence versus locomotion. Internal correlations are highest during locomotion but drop during quiescence. b, Schematic of RSP dynamics during quiescence versus locomotion. Internal correlations are highest during quiescence but drop during locomotion, and units become more strongly affiliated with outside areas, including V1 and SC. 